\title{
Development of a 2D Axisymmetric Electron Fluid Model in Hall Thrusters
}

\author{
Horatiu C. Dragnea* \\ University of Michigan, Ann Arbor, MI, 48109, USA \\ Kentaro $\mathrm{Hara}^{\dagger}$ \\ Texas A\&M University, College Station, TX, 77843, USA \\ Iain D. Boyd ${ }^{\ddagger}$ \\ University of Michigan, Ann Arbor, MI, 48109, USA
}

\begin{abstract}
Modeling and simulation play an important role in the development of Hall thrusters by providing faster and more cost-effective means of characterization, as opposed to using experimental measurements alone. While the hybrid-PIC approach has been well established for the past two decades, new thruster designs require updates in the computational models. Specifically, the quasi-1D fluid electron model places significant limitations on the simulation, and this study focuses on the development of a $2 \mathrm{D}$-axisymmetric electron model. First, the potential solver is verified by proving current conservation for all seven test cases. Next, the model is benchmarked against a quasi-1D model to evaluate discharge current, plasma potential, axial electric field, global Joule heating, and Joule heating power density. For the three channel test cases with a purely radial electric field the domain is the most similar to the quasi-1D domain, and we see the best agreement with the quasi-1D results. The difference in electron current is less than $1 \%$, and good qualitative agreement is observed for the plasma potential, axial electric field and Joule heating power density. For the three channel test cases with a curved magnetic field we observe a difference in discharge current of less than $10 \%$, while for the plume case the current is $70 \%$ higher than in the quasi-1D prediction, due to the location and geometry of the boundary conditions. Finally, the effects of Joule heating are investigated, and high sensitivity to the electron collision frequency and magnetic field is observed. While the present work highlights the latest developments of a complete standalone $2 \mathrm{D}$ model for electrons, ongoing work is focused on coupling this model with a heavy species solver in a hybrid framework.
\end{abstract}

\section{Nomenclature}

$\vec{B}$
$\vec{E}$
$e$
$j_{e}, j_{i}$
$k_{r}, k_{z}$
$m_{e}$
$n_{e}$
$p$

magnetic field vector
electric field vector
elementary charge
electron and ion current density
radial and axial index
electron mass
electron number density
electron pressure

$$
\begin{aligned}
& T_{e} \\
& \Delta r, \Delta z \\
& \mu \\
& \nu_{e} \\
& \phi \\
& \Gamma_{e, r}, \Gamma_{e, z}
\end{aligned}
$$

electron temperature

radial and axial spatial step size electron mobility

total effective electron collision frequency plasma potential radial and axial electron fluxes

\footnotetext{
*PhD Candidate, Department of Aerospace Engineering, Ann Arbor, MI, 48109, USA, AIAA Student Member.

$\dagger$ Assistant Professor, Department of Aerospace Engineering, College Station, TX, 77843, 08543, USA, AIAA Member

${ }_{\ddagger}^{\ddagger}$ James E. Knott Professor, Department of Aerospace Engineering, Ann Arbor, MI, 48109, USA, AIAA Fellow.
} 


\section{Introduction}

Hall effect thrusters (HETs) have a long history of over five decades of development and use on spacecraft for stationkeeping and orbit-raising. While experimental testing is crucial for flight qualification and scientific investigations, it is expensive, and in some aspects limited. A complementary tool that helps characterize Hall thrusters is modeling and simulation.

The hybrid-particle-in-cell (hybrid-PIC) framework HPHall-3 has been used to model many thrusters, and is considered a standard for simulation. It was developed by Fife ${ }^{1}$ and updated several times ${ }^{2-5}$. The heavy species physics is resolved using particles, which ensures that non-Maxwellian velocity distribution functions (VDFs) may be captured. Although PIC produces noisy number density results compared to a fluid model ${ }^{5}$ , the number of simulation macro particles may be increased to improve the resolution. An advantage of the PIC solver is the ability of HPHall to capture the breathing mode oscillations that occur in $\mathrm{HETs}^{6}$. Due to the small mass of electrons, the computational costs may become prohibitively expensive if they are modeled as particles, thus a fluid approach is used in an effort to reduce computational time. In addition, the thermalized potential approximation is employed to further reduce the dimensionality of the problem. The electron temperature is assumed constant along the magnetic field lines, and this forms the basis of the quasi-1D electron model. However, this approach is inadequate for modeling more complicated magnetic field topologies, as seen in magnetically shielded HETs ${ }^{7}$ or nested channel devices ${ }^{8,9}$.

Therefore, a 2D axisymmetric electron model is developed to replace the quasi-1D model that is currently used in HPHall. The physical model is described in Section II: first the potential equation in Section A, followed by a presentation of the energy conservation equation in Section B, and the numerical implementation is described in Section C. A first comparison with the quasi-1D model was presented in a previous study ${ }^{10}$ , where results from two test cases and two thrusters were evaluated, and in the present work we show further verification and benchmarking of the model while also describing the new capabilities which include simulating the plume, and the addition of an energy equation solver. The new test cases are described in Section III, and the results are discussed in Section IV. Section V summarizes the conclusions of this study. Finally, Section VI describes the future development of the model, and its application to study HETs.

\section{Physical Model}

The goal is to model the momentum (Section A) and energy (Section B) conservation for electrons in a

fluid formulation. In order to simplify the equations in the upcoming sections we introduce four coefficients in Eqs. 2-5, based on the electron mobility (Eq. 1) and magnetic field.

$$
\begin{aligned}
\mu & =\frac{e}{m_{e} \nu_{e}} \\
\mu_{z z} & =\left(\frac{1+\mu^{2} B_{z}^{2}}{1+\mu^{2} B^{2}}\right) \\
\mu_{z r} & =\left(\frac{-\mu B_{\theta}+\mu^{2} B_{z} B_{r}}{1+\mu^{2} B^{2}}\right) \\
\mu_{r z} & =\left(\frac{\mu B_{\theta}+\mu^{2} B_{z} B_{r}}{1+\mu^{2} B^{2}}\right) \\
\mu_{r r} & =\left(\frac{1+\mu^{2} B_{r}^{2}}{1+\mu^{2} B^{2}}\right)
\end{aligned}
$$

\section{A. Potential}

Following a similar procedure as Geng ${ }^{11}$ and Koo, ${ }^{12}$ we start with the Generalized Ohm's Law. Considering the electron fluxes by components, we may write Eq. 6 for the axial flux, and Eq. 7 for the radial flux. 


$$
\begin{aligned}
& \Gamma_{e, z}=n_{e} \mu_{z z} E_{z}+n_{e} \mu_{z r} E_{r}+\mu_{z z} \frac{\partial p}{\partial z}+\mu_{z r} \frac{\partial p}{\partial r} \\
& \Gamma_{e, r}=n_{e} \mu_{r z} E_{z}+n_{e} \mu_{r r} E_{r}+\mu_{r z} \frac{\partial p}{\partial z}+\mu_{r r} \frac{\partial p}{\partial r}
\end{aligned}
$$

Further considering charge conservation, and assuming quasi neutrality, we arrive at the governing equation for plasma potential:

$$
\begin{aligned}
L H S & =\frac{\partial}{\partial z}\left(n_{e} \mu_{z z} \frac{\partial \phi}{\partial z}+n_{e} \mu_{r z} \frac{\partial \phi}{\partial r}\right)+\frac{\partial}{\partial r}\left(n_{e} \mu_{z r} \frac{\partial \phi}{\partial z}+n_{e} \mu_{r r} \frac{\partial \phi}{\partial r}\right) \\
& +\left(\frac{n_{e} \mu_{z r} \frac{\partial \phi}{\partial z}+n_{e} \mu_{r r} \frac{\partial \phi}{\partial r}}{r}\right) \\
R H S & =\frac{\partial}{\partial z}\left(\mu_{z z} \frac{\partial p}{\partial z}+\mu_{r z} \frac{\partial p}{\partial r}\right)+\frac{\partial}{\partial r}\left(\mu_{z r} \frac{\partial p}{\partial z}+\mu_{r r} \frac{\partial p}{\partial r}\right) \\
& +\frac{\mu_{z r} \frac{\partial p}{\partial z}+\mu_{r r} \frac{\partial p}{\partial r}}{r}-\frac{1}{e}\left(\frac{\partial j_{i, r}}{\partial r}+\frac{\partial j_{i, z}}{\partial z}+\frac{j_{i, r}}{r}\right)
\end{aligned}
$$

\section{B. Energy}

We start with the energy equation in vector form used in Hall2De $\mathrm{De}^{13,14}$ :

$$
\begin{aligned}
\frac{3}{2} e n_{e} \frac{\partial T_{e}}{\partial t} & =\vec{E} \cdot \vec{j}_{e}+\nabla \cdot\left(\frac{5}{2} T_{e} \vec{j}_{e}+\underline{\underline{\kappa}}_{e} \cdot \nabla T_{e}\right)-\frac{3}{2} T_{e} \nabla \cdot \vec{j}_{e} \\
& -\sum_{s} \dot{n}_{s} e\left(\epsilon_{s}+\frac{3}{2} T_{e}\right)+Q_{e}^{T}
\end{aligned}
$$

where $e$ is the elementary charge, $n_{e}$ is the electron number density, $T_{e}$ is the electron temperature, $\vec{E}$ is the electric field $\vec{j}_{e}$ is the electron current density, $\underline{\underline{\kappa}}_{e}$ is the electron thermal conductivity tensor, $\dot{n}_{s}$ is the electron impact-ionization rate for species $s, \epsilon_{s}$ is the ionization potential of species $s$ and $Q_{e}^{T}$ is the electron thermal heat-exchange term ${ }^{14}$ defined in Eq. 11.

$$
Q_{e}^{T}=-\sum_{s} n_{e} \frac{2 m_{e}}{m_{s}} \nu_{e s} \frac{3}{2}\left(\frac{k T_{e}}{e}-\frac{k T_{s}}{e}\right)
$$

where $\nu_{e s}$ is the collision frequency between electrons and species $s$.

For the initial implementation we neglect the collision and ionization terms, and expand all other terms. After simplifications, we write a master equation for the energy equation that applies to the axisymmetric coordinate system: Eq. 12. Note that while for the momentum equation there is no time dependent term due to the assumption of negligible electron inertia, in the energy equation we are left with a time derivative of temperature. 


$$
\begin{aligned}
\frac{3}{2} e n_{e} \frac{\partial T_{e}}{\partial t} & =E_{r} \cdot j_{e r}+E_{z} \cdot j_{e z} \\
& +T_{e} \cdot\left[\left(\frac{\partial j_{e r}}{\partial r}\right)+\frac{j_{e r}}{r}+\left(\frac{\partial j_{e z}}{\partial z}\right)\right] \\
& +j_{e r} \cdot\left(\frac{\partial T_{e}}{\partial r}\right)+j_{e z} \cdot\left(\frac{\partial T_{e}}{\partial z}\right) \\
& +\left[\frac{\kappa_{r}}{r}\left(\frac{\partial T_{e}}{\partial r}\right)+\kappa_{r} \frac{\partial^{2} T_{e}}{\partial r^{2}}+\left(\frac{\partial T_{e}}{\partial r}\right)\left(\frac{\partial \kappa_{r}}{\partial r}\right)\right. \\
& \left.+\kappa_{z} \frac{\partial^{2} T_{e}}{\partial z^{2}}+\left(\frac{\partial T_{e}}{\partial z}\right)\left(\frac{\partial \kappa_{z}}{\partial z}\right)\right]
\end{aligned}
$$

\section{Numerical Implementation}

A flowchart of the electron model is shown in Fig. 1. The first box shows the inputs: the thruster magnetic field, which is constant in time, the electron mobility, and the electron number density which due to the quasi-neutrality assumption is derived from a heavy-species calculation. Next an initial guess is made for the electron temperature. For the present work, these values are HPHall time-averaged results. The potential solver algorithm is then applied to compute the plasma potential, electric field and electron current density. These values are then used in the temperature solver. If the number of maximum electron timesteps $\left(N_{e}\right)$ is reached, then the plasma potential, electric field, electron current density and electron temperature are output. Otherwise, the process is repeated with the new value of electron temperature.

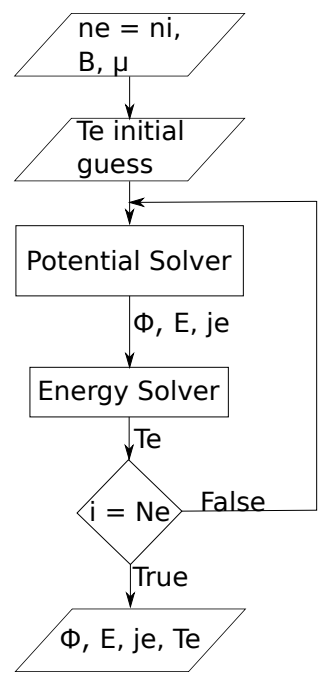

Figure 1. Electron model flowchart.

The physical equations described in Section II are discretized using a conservative 9-point finite-difference scheme. This guarantees that the cross-term derivatives $\left(\frac{\partial}{\partial r} \frac{\partial}{\partial z}\right)$ are also included in the computation, and not frozen as in previous models ${ }^{11}$. The stencil requires information from eight neighbors to compute the potential value at the center point. Both plasma potential and electron temperature are computed at the cell center, however, some of the coefficient values are needed on the grid interfaces, which requires the use of staggered grids.

Since the electron inertia is neglected from the start of the theoretical development in the momentum equation, there is no time dependence for the potential solver, so the numerical problem is elliptic, and we are only concerned with a two dimensional spatial discretization. Considering the assumption of Hall thruster axial symmetry, and the handling of derivatives in cylindrical coordinates, the azimuthal direction is implicitly 
accounted for. However, the energy equation must be discretized in both time and space. A semi-implicit Forward-Euler scheme is used for time-integration where the conduction term is computed at the future time-step.

\section{Setup for Test Cases}

Table 1. Test Cases

\begin{tabular}{l|c|c|c} 
Case & B shape & B magnitude & includes plume \\
\hline 1 & radial & low & no \\
2 & radial & medium & no \\
3 & radial & high & no \\
4 & curved & low & no \\
5 & curved & medium & no \\
6 & curved & high & no \\
7 & curved & - & yes
\end{tabular}

In order to evaluate the new 2D electron model, a series of test cases are prepared. Table 1 summarizes the characteristics of each of the seven cases: the first six cases only focus on the thruster channel, and they were developed to reduce differences between the 2D electron model and the HPHall simulation that may be due to mesh or boundary condition handling, in particular the implementation of the cathode line from HPHall $^{10}$. The seventh test case includes the thruster plume. The first three test cases have a zero axial component of the magnetic field, while the final four have a curved magnetic field with both axial and radial nonzero magnetic field components. For the thruster channel cases (1-6) three magnetic field magnitudes are tested: a nominal case (low), an intermediate case, and a high value case (approximately double the value of the low field case, when comparing magnitudes at the location of maximum field strength). The magnetic field topologies are shown in Figure 2. Notice that for the plume case the curvature of the magnetic field is reversed in the plume.

The plasma potential boundary conditions for the channel and plume test cases are shown in Figures 3(a) and 3(b), respectively. The anode boundary is a Dirichlet condition where the potential is set to $300 \mathrm{~V}$, while for the cathode, the value is set to $0 \mathrm{~V}$. The walls, thruster pole pieces, as well as north and south plume boundaries use a dielectric boundary condition for plasma potential. Through this boundary condition we ensure that the electron current equals the ion current at the wall, in the normal direction.

For electron temperature, the anode and cathode use Dirichlet conditions, with values of $2 \mathrm{eV}$ and $20 \mathrm{eV}$, respectively, which are chosen based on time-averaged HPHall results for electron temperature. For the walls, a Neumann type condition is implemented, and the temperature flux is set to zero in the normal direction. No special considerations are included for the tangential direction.

\section{Results and Discussion}

The test cases described in Section III are used to verify the model implementation. As shown in the algorithm flowchart from Fig. 1, the plasma potential solver requires input values of magnetic field, electron mobility, number density and temperature. In order to obtain these values, we first prepare a set of HPHall simulations, and use the time-averaged output data as inputs for the $2 \mathrm{D}$ electron model. The HPHall simulation data serves a dual purpose: it is also used for comparison with values computed by the 2D electron model. All results shown from the 2D electron model are obtained after a single electron timestep.

Before comparing the 2D and quasi-1D electron models, it is important to understand the difference between the two domains, and thus we present the spatial distribution of Hall parameter for Case 4 in Figure 4 . We

observe null values of Hall parameter near the cathode, where we would expect to see a maximum. The 


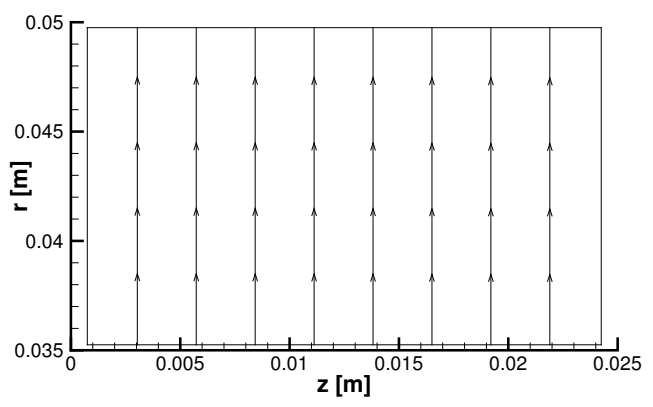

(a) Cases 1, 2, and 3 .

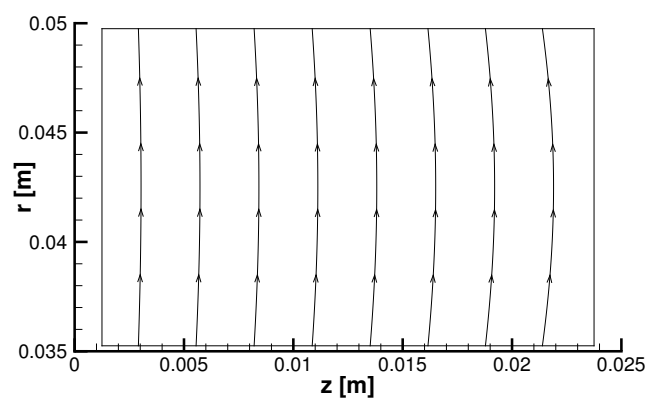

(b) Cases 4, 5, and 6 .

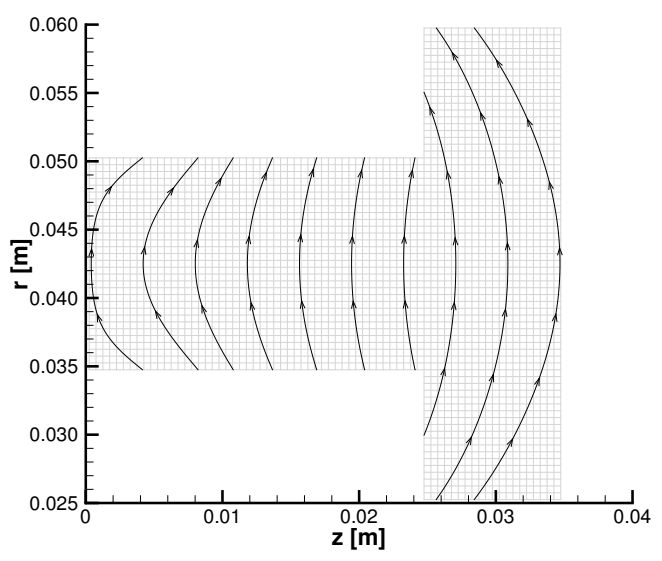

(c) Case 7 .

Figure 2. Magnetic field topologies. 

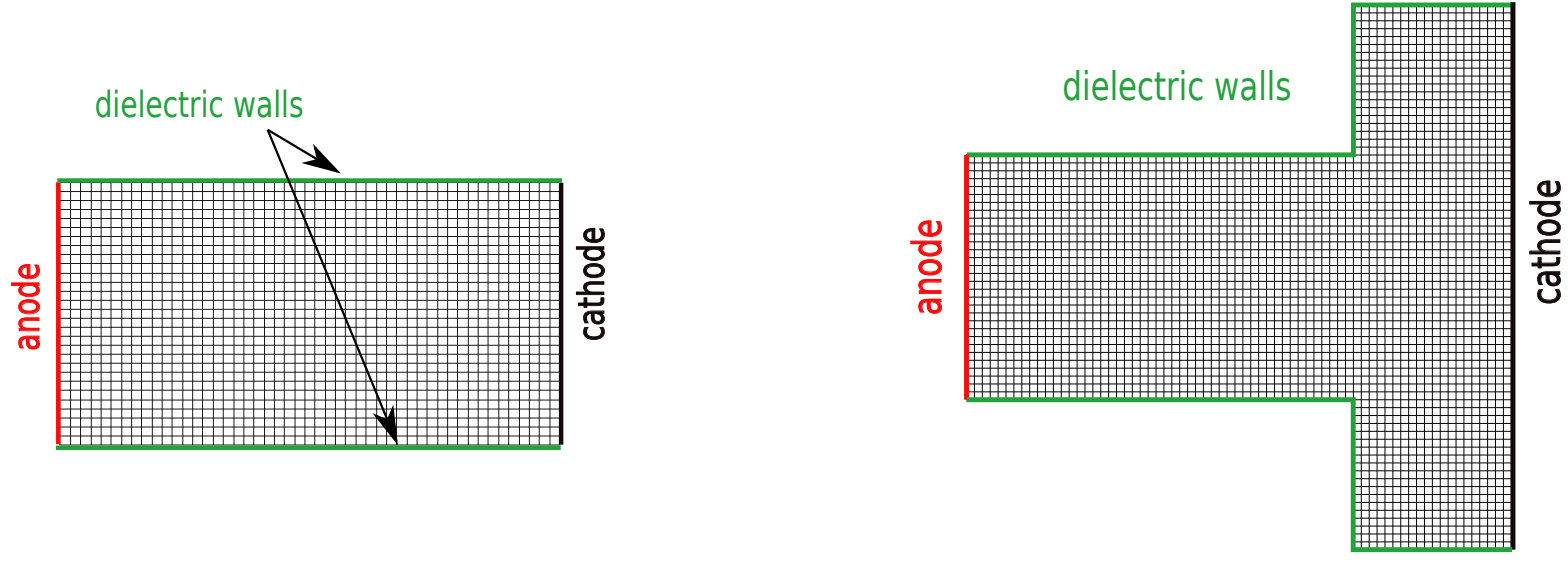

(a) Cases 1-6.

(b) Case 7 .

Figure 3. Domain and boundary condition types for potential. 
Joule heating computation is sensitive to the values of the electron collision frequency and the magnetic field, and based on the plots of Hall parameter, in the region of the domain where the magnetic field dominates, HPHall does not include the appropriate terms in the quasi-1D computational domain. While within the 2D model we compute the electron collision frequency for the regions omitted by the quasi-1D domain, other parameters such as the initial value of electron temperature may be responsible for significant differences between the two models.
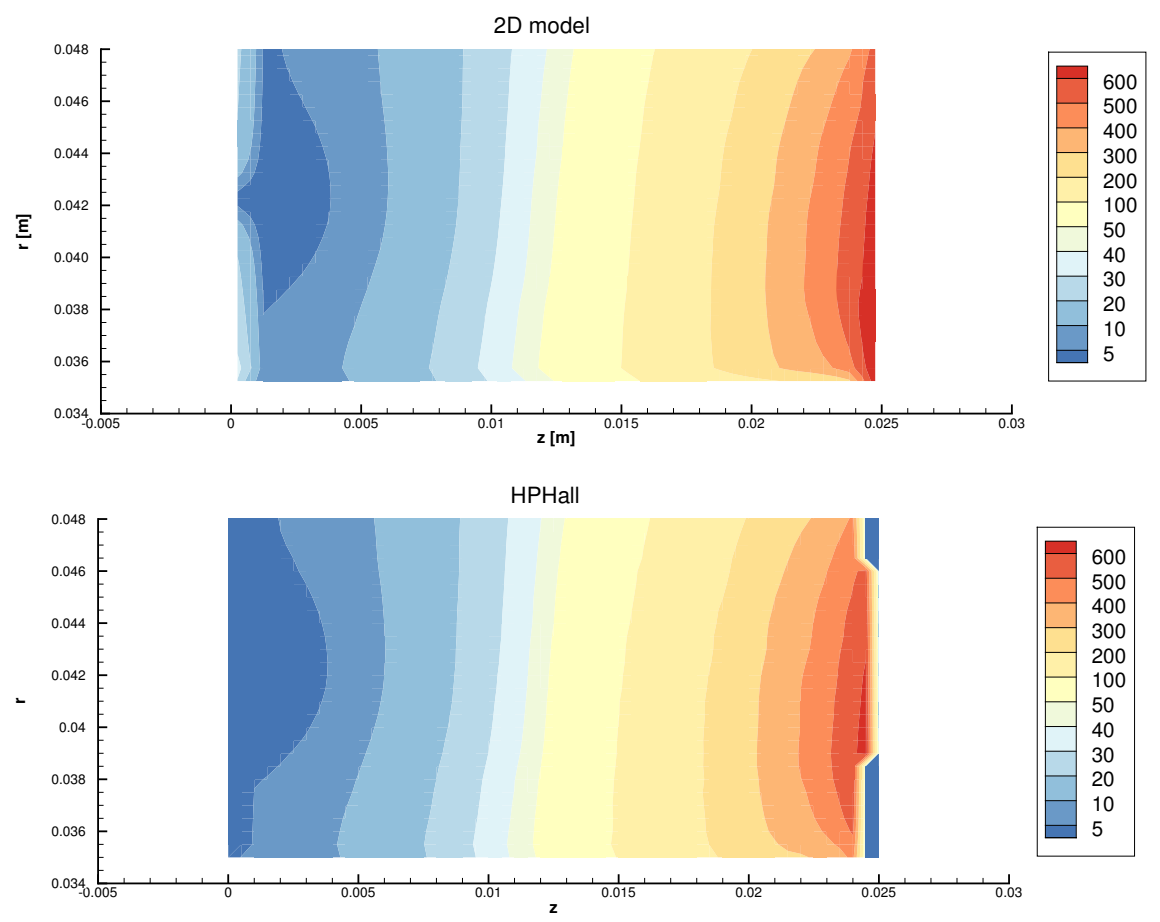

Figure 4. Hall parameter.

\section{A. Electron Current}

The first step for model verification is the computation of the electron current at the anode and cathode. These values are calculated by integrating the electron current density radially at the anode and cathode locations. In Table 2 we observe that in all five test cases the electron current is conserved through the domain. While for simulation results we typically report data with three significant digits, in the present case the anode and cathode currents were identical up to the fourth decimal place, suggesting that maximum losses due to the finite discretization are only $0.00049 \%$.

Table 2. Discharge Current

\begin{tabular}{l|c|l|l|r} 
Case & $\mathrm{B}_{\max }(\mathrm{G})$ & $\mathrm{I}_{\text {anode }}(\mathrm{A}), 2 \mathrm{D}$ & $\mathrm{I}_{\text {cathode }}(\mathrm{A}), 2 \mathrm{D}$ & $\mathrm{I}_{d}(\mathrm{~A})$, quasi-1D \\
\hline 1 & 317 & 4.16 & 4.16 & 4.16 \\
2 & 422 & 3.40 & 3.40 & 3.37 \\
3 & 633 & 2.49 & 2.49 & 2.47 \\
4 & 316 & 4.56 & 4.56 & 4.50 \\
5 & 422 & 3.59 & 3.59 & 3.42 \\
6 & 633 & 2.36 & 2.36 & 2.18 \\
7 & 790 & 5.03 & 5.03 & 2.96
\end{tabular}


Next we compare the current computed from the 2D electron model with the time-averaged discharge current that was output from HPHall. The values match to within $1 \%$ for cases 1, 2 and 3 . For the curved cases we observe a larger difference when compared to the quasi-1D results, however all values are within 10\%: $1.4 \%$, $5 \%$ and $8.3 \%$ for cases 4,5 and 6 respectively. This discrepancy may be due to the differences observed between the quasi-1D and 2D computational domains. In HPHall the computational domain for electrons lies between the anode and cathode magnetic field lines, however, due to the curvature of the field there will be regions outside the quasi-1D domain that are part of the $2 \mathrm{D}$ domain.

Finally, the largest difference $(70 \%)$ is observed for Case 7 . Here the quasi-1D current is significantly lower due to the boundary differences between the two simulations. While in HPHall the mesh extends further into the plume and ends with a curved cathode line, in the $2 \mathrm{D}$ model, the straight cathode boundary is encountered further upstream. While in principle the 2D formulation places no restrictions on the size of the computational domain, and how far it can extend into the plume, at the present development stage of the model there is a practical barrier. Output data from HPHall is used as input for the 2D model therefore the domain of the 2D model must be circumscribed within the quasi-1D domain. This limitation will be eliminated once the 2D electron model is fully integrated within the hybrid framework, however while it is still a standalone model, the domain size remains a limiting factor.

\section{B. Plasma Potential}

Next, we consider the plasma potential spatial distribution for Cases 1-6, as shown in Fig. 5 from both the 2D computation (top), and HPHall (bottom). The radial and curved magnetic field cases are shown side-byside for corresponding fields strengths. Good qualitative agreement is observed between the $2 \mathrm{D}$ channel test cases and the quasi-1D results. We observe a non-physical decrease of the potential in the HPHall simulation near the anode (the value should be $300 \mathrm{~V}$ ). A similar problem was encountered in a previous benchmarking effort with HPHall, ${ }^{10}$ and it was concluded that it is due to the qusi-1D domain not extending all the way to the anode. In the 2D model the value is imposed exactly at the west cell interface, which is why we notice some differences in the near-anode region.

Figure 6 shows the plasma potential distribution for the plume test case. The effects of the dielectric boundary condition are visible in the near-field plume where a larger plasma potential value is maintained in the 2D model output than in the quasi-1D result. Since we are using input data from HPHall, the size of the $2 \mathrm{D}$ model domain is limited to the quasi-1D domain. A larger plume domain may provide a larger radial distance for the plasma potential to diffuse, which may lead to a more rapid decrease, similar to what we are seeing in the HPHall output. In the following sections we will only focus on the channel test cases, leaving the plume analysis for future work.

\section{Axial Electric Field}

The axial electric field is responsible for accelerating the ions in a HET, thus having the largest contribution in thrust production. In Figure 7 we show spatial distributions of the axial electric field for the channel cases (1-6). When comparing to HPHall, we observe good agreement inside the thruster channel for all test cases, except for the cathode region. This is the area of maximum electric field, and due to differences between the quasi-1D and 2D domain size we observe large differences in the electric field in this region.

\section{Joule Heating}

Due to the limitations explained above regarding the domain size and the need to use HPHall data for an input, the electron temperature solver is first tested only in the channel region. Since the electric fields and electron current densities are high within the thruster channel, we expect that the Joule heating term $\vec{E} \cdot \vec{j}_{e}$ will play a significant role in the energy equation, so we focus on this term.

A global value of Joule heating power is computed by integrating Eq. 13 in cylindrical coordinates, as shown in Fig. 8 to obtain Eq. 14. In Table 3 we report the global values of Joule heating (Watts) obtained from 

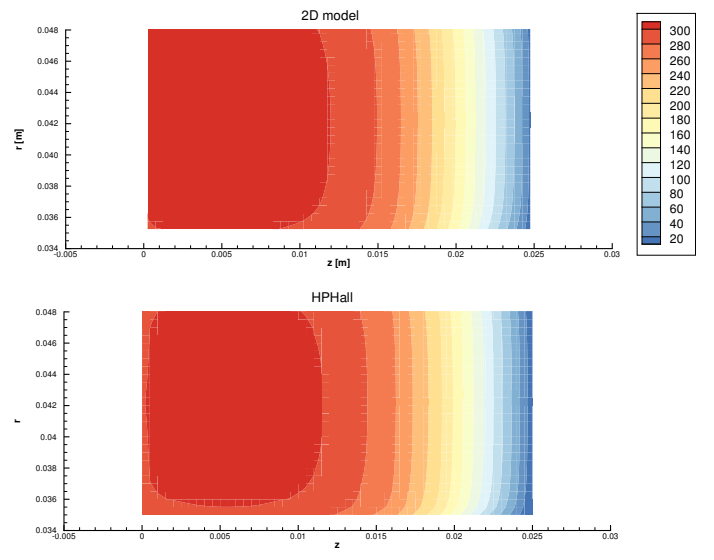

(a) Case 1 .
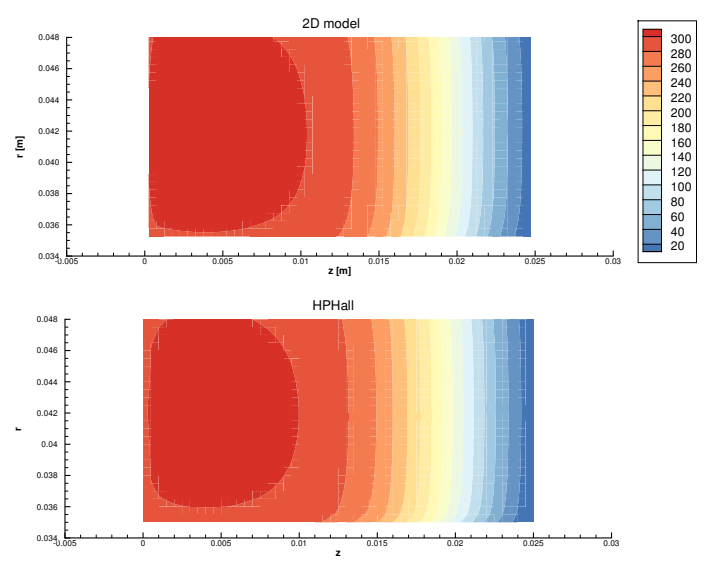

(c) Case 2 .
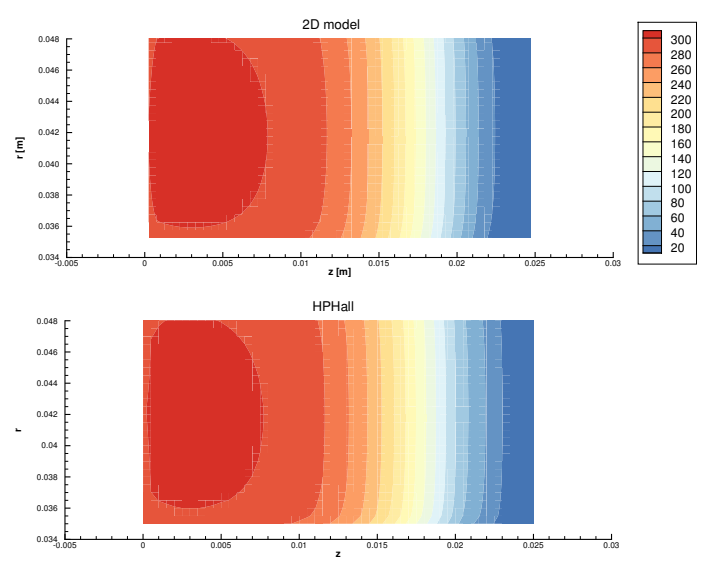

(e) Case 3 .
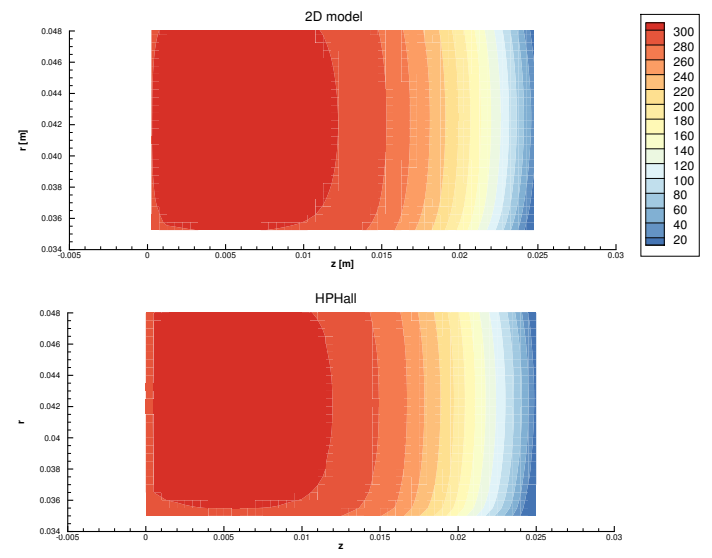

(b) Case 4 .
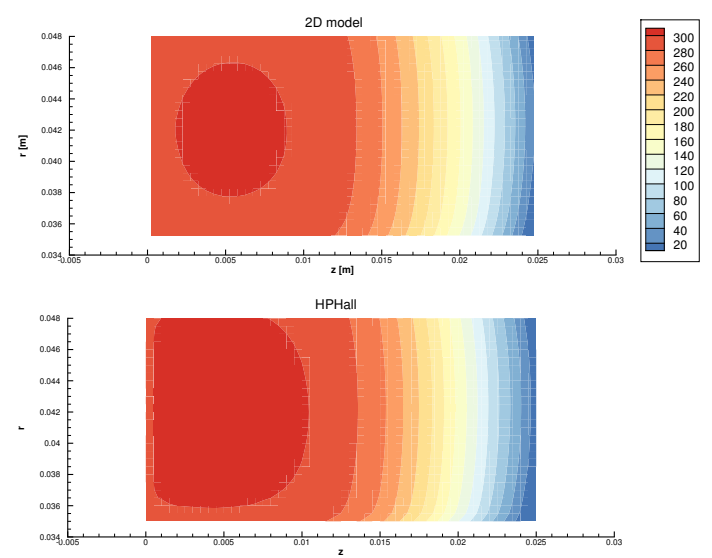

(d) Case 5 .
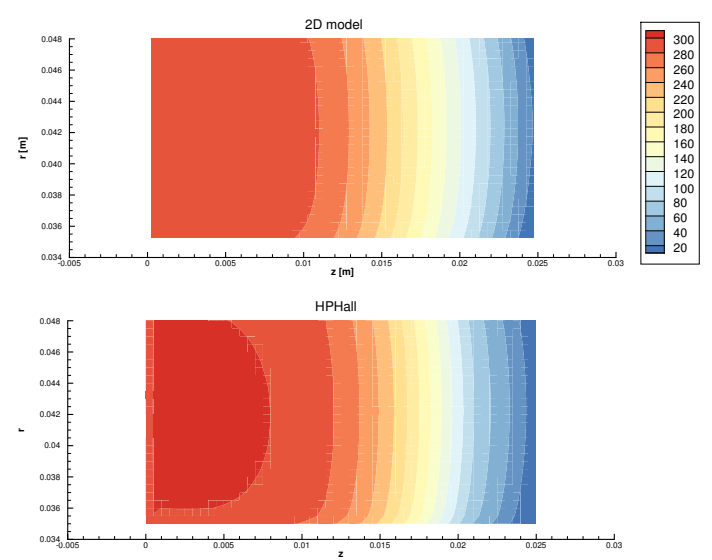

(f) Case 6 .

Figure 5. Plasma potential. 

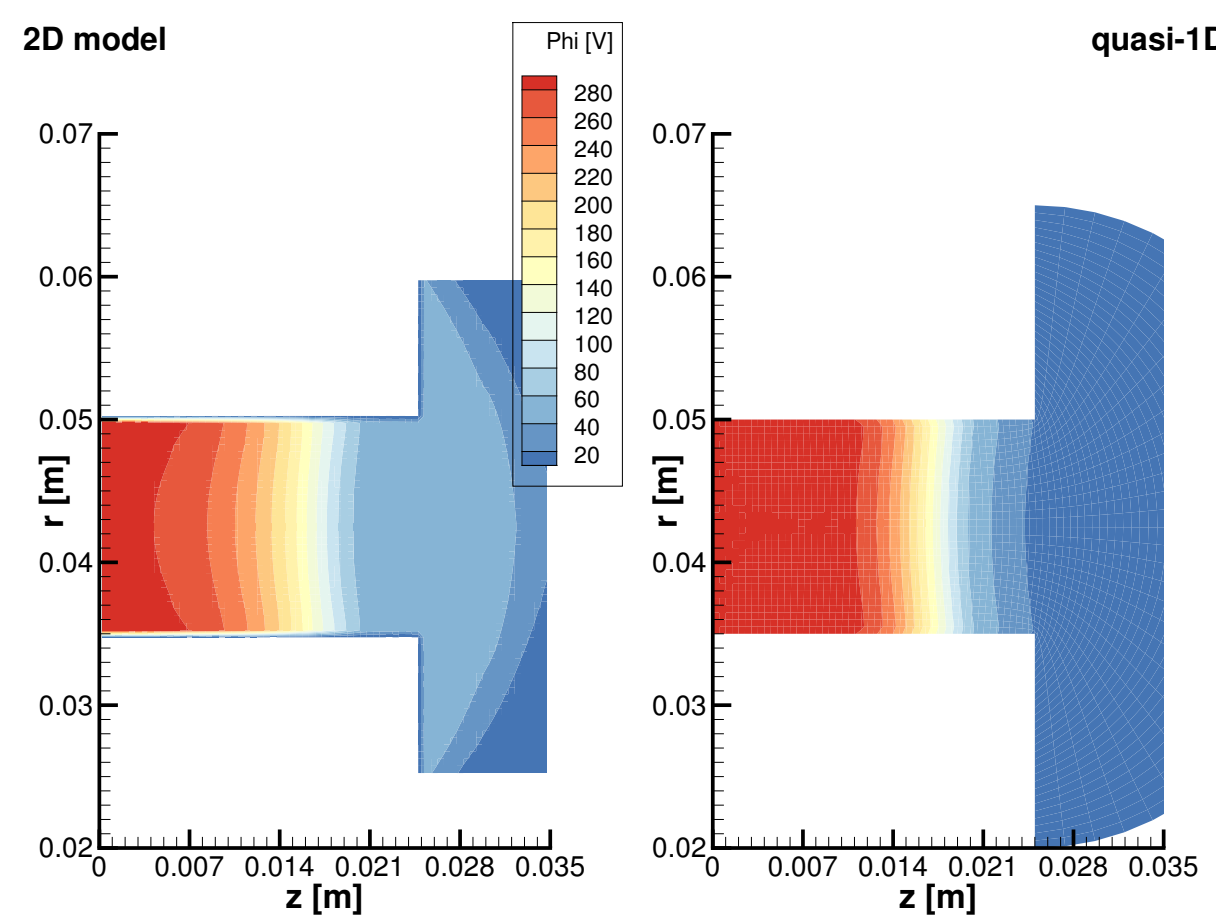

Figure 6. Case 7.

both the 2D electron model, and the quasi-1D model in HPHall, while Figure 9 shows the spatial distribution of Joule heating power density (Watts per meter cubed).

$$
\begin{aligned}
& p_{\text {heating }}=E_{r} \cdot j_{e, r}+E_{z} \cdot j_{e, z} \\
& P_{\text {heating }}=\iiint p_{\text {heating }}(r, z) d z\left[\pi\left(R_{2}^{2}-R_{1}^{2}\right)\right]
\end{aligned}
$$

We note here that in HPHall there is a single computational dimension, and $j_{e, r}$ and $j_{e, z}$ are computed by projecting the normal electron current onto the axial and radial coordinate system, as shown in Eq. 16.

$$
\begin{aligned}
& j_{e, z}=j_{e} \cdot \frac{B_{r}}{B} \\
& j_{e, r}=j_{e} \cdot \frac{B_{z}}{B}
\end{aligned}
$$

However, in the 2D model we compute the current in each direction from the electron flux expressions presented earlier in Eq. 6 and 7:

$$
\begin{aligned}
j_{e, z} & =-e \cdot \Gamma_{e, z} \\
j_{e, r} & =-e \cdot \Gamma_{e, r}
\end{aligned}
$$

Thus, for the purely radial cases, in HPHall we observe a null radial electron current, while the 2D code will provide a nonzero value. This fundamental difference is responsible for the discrepancies observed in the Joule heating values for Cases 1, 2 and 3 .

The values of Joule heating are within an order of magnitude of the discharge power for all cases. We observe closer agreement between the 2D model and HPHall for the cases with a purely radial magnetic field, than 

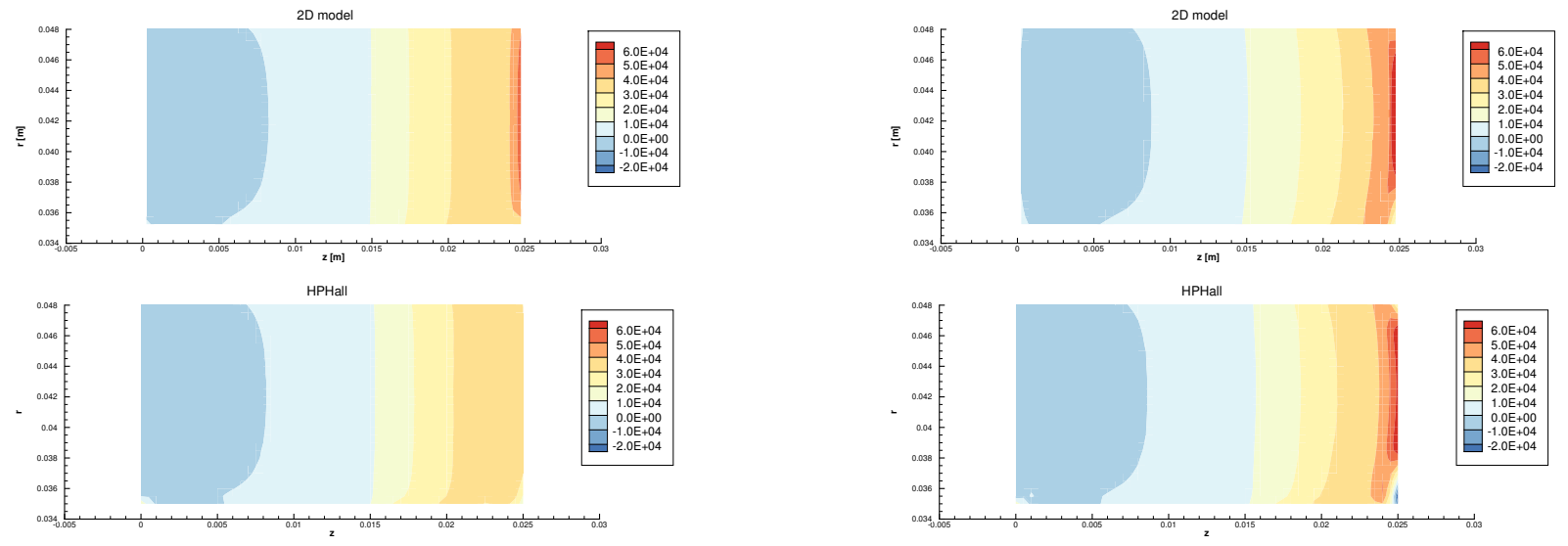

(a) Case 1 .

(b) Case 4 .
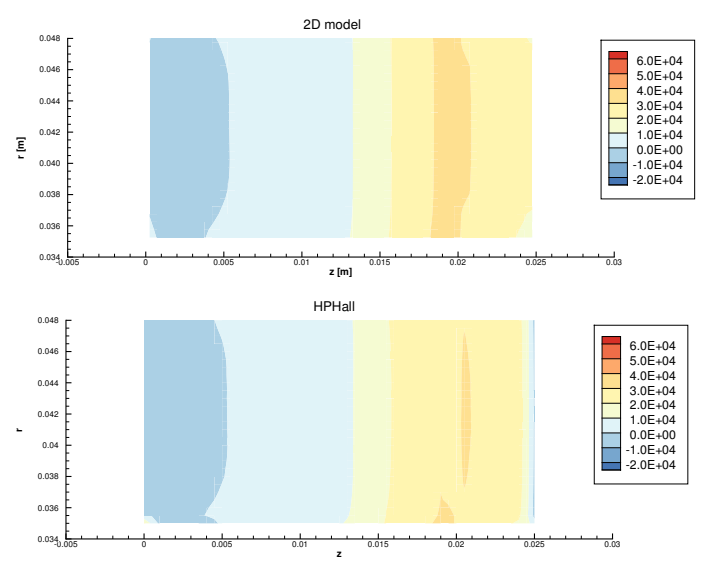

(c) Case 2 .
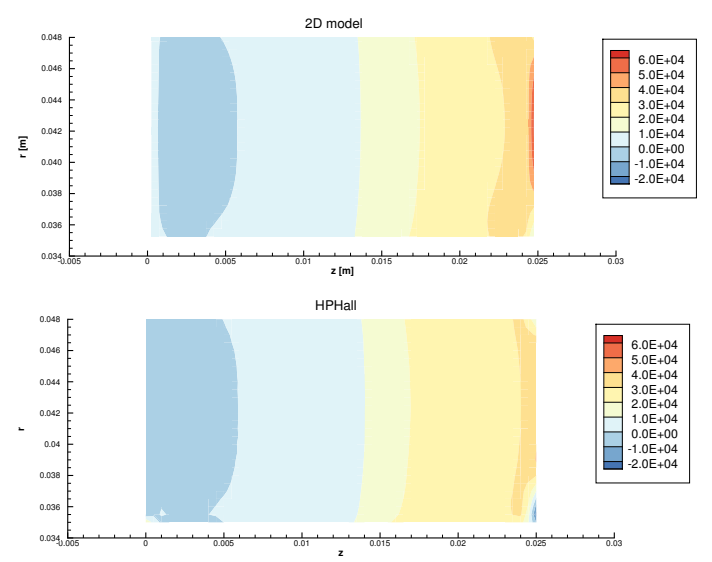

(d) Case 5 .
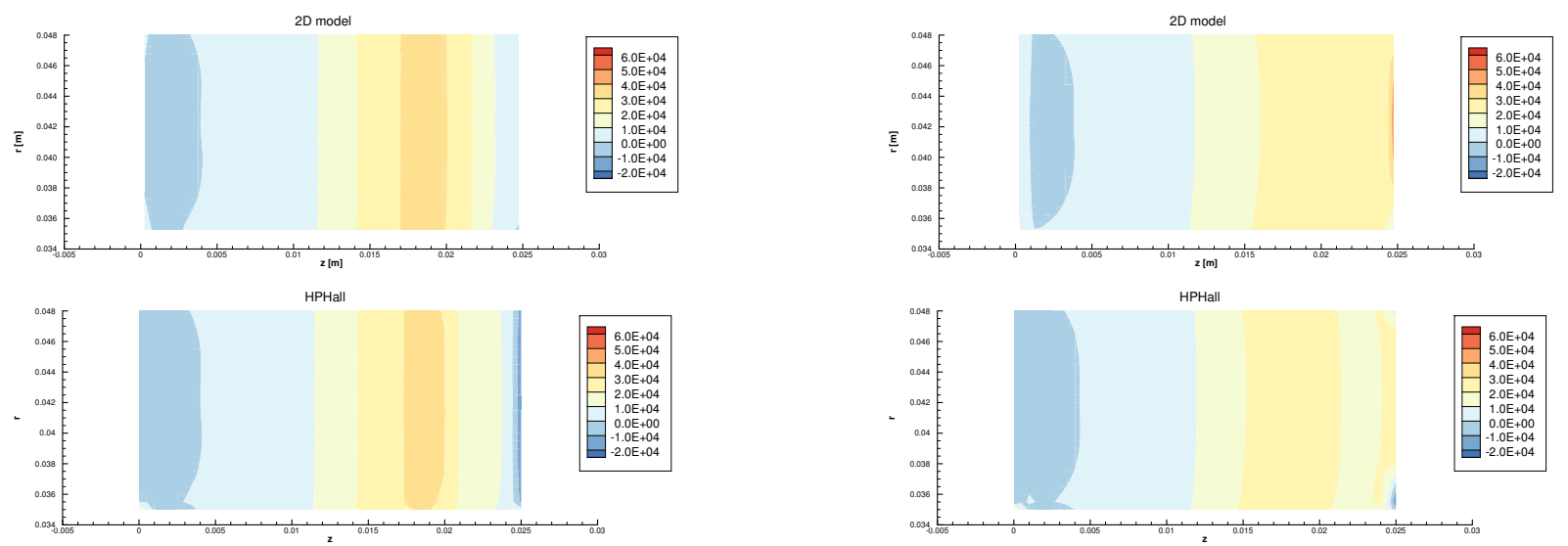

(e) Case 3 .

(f) Case 6 .

Figure 7. Axial electric field 


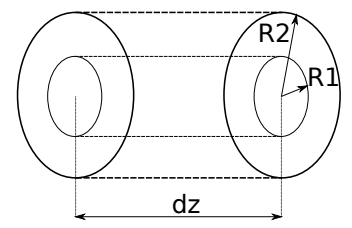

Figure 8. Schematic for cylindrical integration.

Table 3. Power comparison

\begin{tabular}{c|c|r|r} 
Case & Method & Discharge Power (W) & Joule heating (W) \\
\hline \hline \multirow{2}{*}{1} & $2 \mathrm{D}$ & 1,250 & 449 \\
& quasi-1D & 1,250 & 323 \\
\hline \multirow{2}{*}{2} & $2 \mathrm{D}$ & 1,020 & 331 \\
& quasi-1D & 1,010 & 282 \\
\hline \multirow{2}{*}{3} & $2 \mathrm{D}$ & 747 & 214 \\
& quasi-1D & 741 & 232 \\
\hline \multirow{2}{*}{4} & $2 \mathrm{D}$ & 1,370 & $-5,330$ \\
& quasi-1D & 1,250 & 367 \\
\hline 5 & $2 \mathrm{D}$ & 1,080 & -588 \\
& quasi-1D & 1,030 & 295 \\
\hline 6 & $2 \mathrm{D}$ & 708 & -48.3 \\
& quasi-1D & 654 & 193
\end{tabular}

those with a curved topology. Thus, for Case 1 we obtain a value that is $39 \%$ higher than the quasi-1D value, for Case 2 the $2 \mathrm{D}$ result is $17 \%$ higher and for Case 3 the $2 \mathrm{D}$ model gives a global Joule heating value that is $7.8 \%$ lower than the quasi-1D output from HPHall. Good qualitative agreement is also observed for the radial magnetic field cases in the heating power density spatial distributions computed with the 2D model and quasi-1D models, as shown in Fig. 9(a), Fig. 9(c) and Fig. 9(e). We observe cooling near the anode region, and heating in the rest of the domain. The largest values of heating power density are seen in the ionization region. Case 3 shows a negative value in the SE corner, while the quasi-1D model does not, however this region is outside the quasi-1D domain, making it difficult to compare results for this portion of the domain.

Meanwhile, the 2D model produces significantly different Joule heating values for the curved magnetic field cases. In addition to magnitude differences ranging between 1.2 (Case 6) and 15 (Case 4), the values computed by the 2D model are negative, while the HPHall values are positive. Further investigation shows large negative regions near the cathode in the heating power density spatial distribution for the $2 \mathrm{D}$ case. In contrast, the HPHall results show a continuous band of positive values. Figures 9(b), 9(d) and 9(f) illustrate the anomalous Joule heating distribution. We observe that as we lower the discharge power (Case 4 - 6), the negative regions become smaller. Since the largest effect is seen for Case 4, we perform a grid sensitivity study for this test case. While the HPHall test case was ran on a $51 \times 31$ mesh, we interpolate the quasi-1D results to a series of meshes to provide a finer grid input to the $2 \mathrm{D}$ model. Figure 10 shows how the mesh refinement is reducing the negative regions near the cathode, and gradually brings the heating power distribution closer to the coarse mesh quasi-1D result (Fig. 10(f)). 

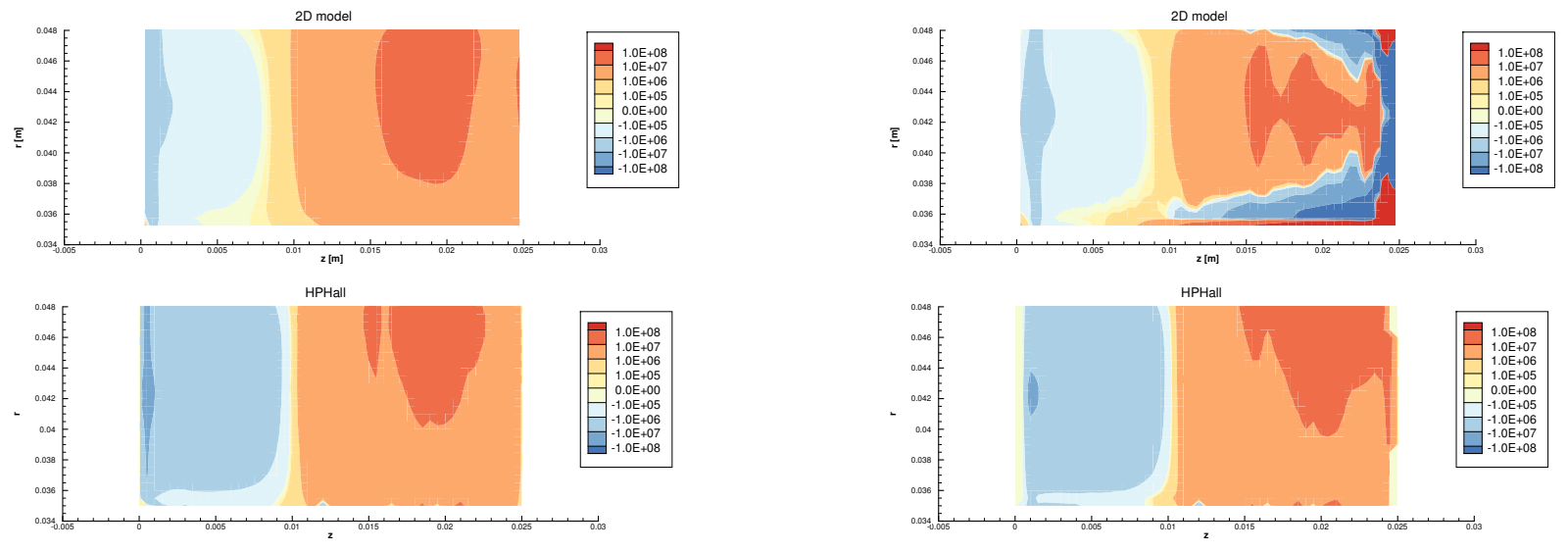

(a) Case 1.

(b) Case 4 .
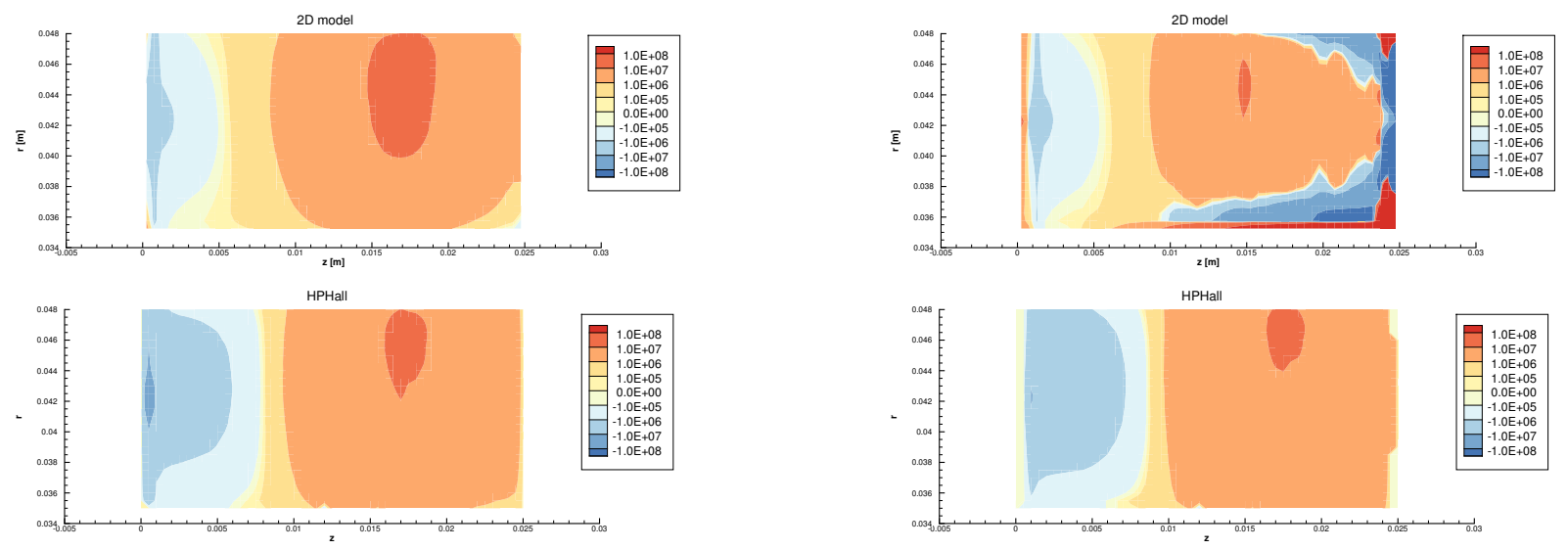

(c) Case 2 .

(d) Case 5 .
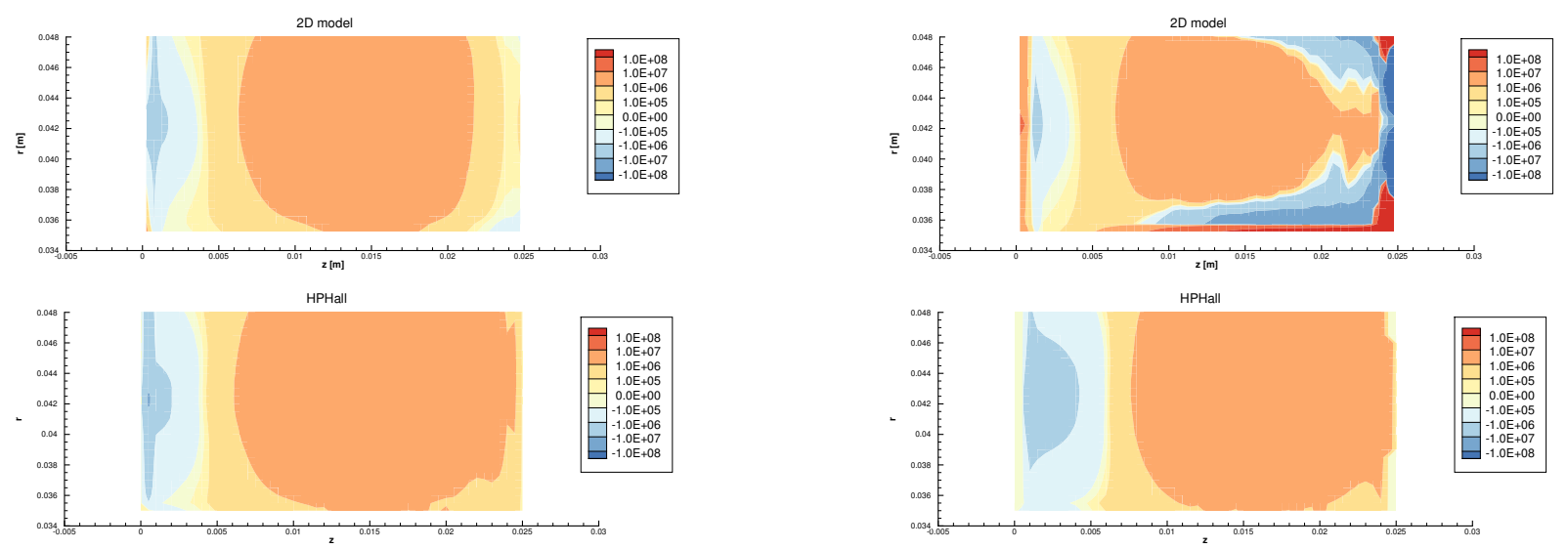

(e) Case 3 .

(f) Case 6 .

Figure 9. Joule heating power density. 


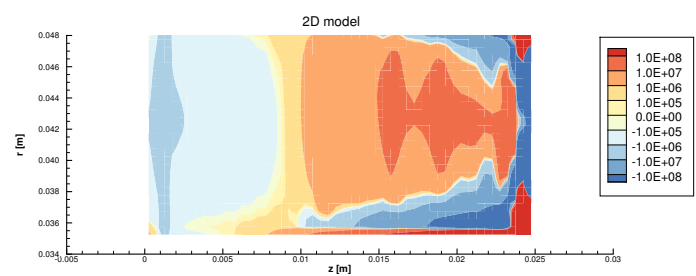

(a) $2 \mathrm{D}, 51 \times 31$.

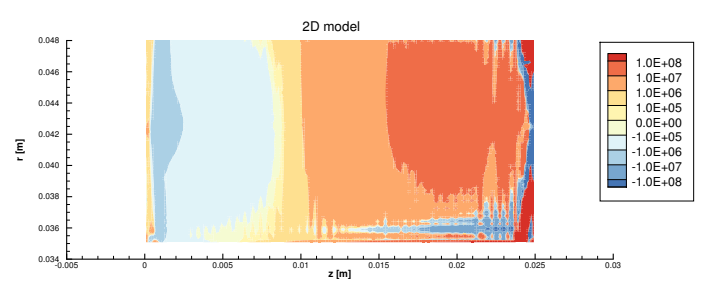

(c) $2 \mathrm{D}, 151 \times 91$.

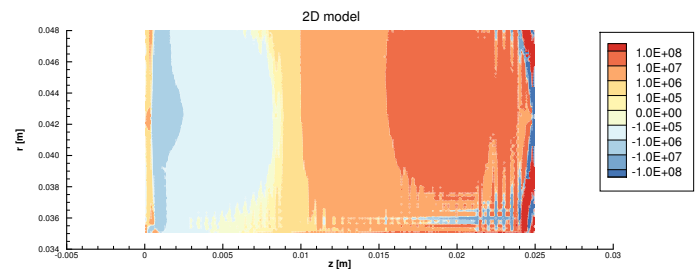

(e) $2 \mathrm{D}, 251 \times 151$.

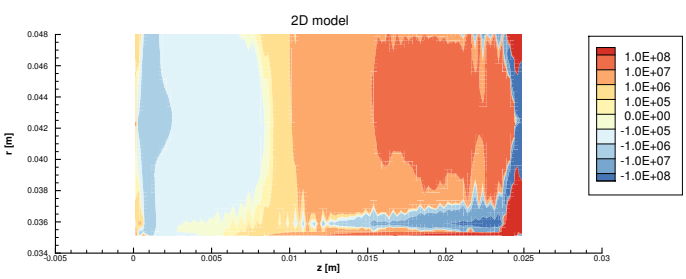

(b) 2D, 101x61.

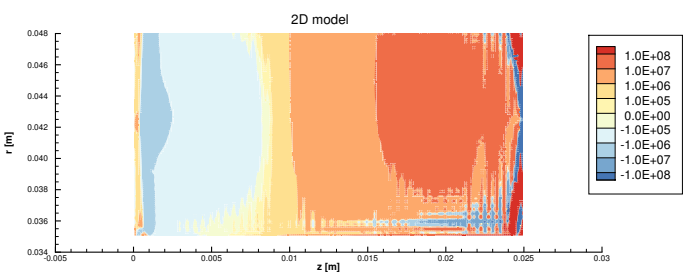

(d) $2 \mathrm{D}, 201 \times 121$.

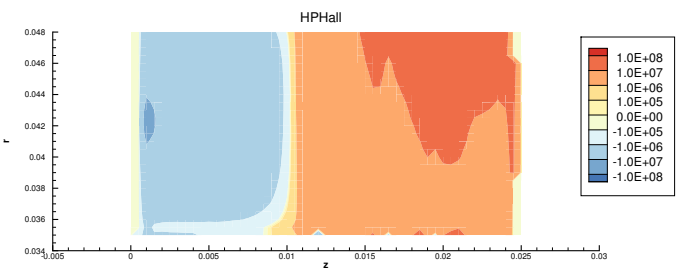

(f) Quasi-1D, 51x31.

Figure 10. Joule heating for Case 4.

\section{Conclusion}

The latest updates in the development of a 2D axisymmetric electron model were presented. The computational domain has been extended to perform plume simulations, and an energy equation solver was implemented. The standalone $2 \mathrm{D}$ electron model was verified, and then benchmarked against a quasi-1D model that was coupled with a PIC code in a hybrid framework (HPHall). The first goal was to verify current conservation by checking the anode and cathode values. Exact current conservation was observed to within three significant figures. Next, the values of electron current were compared to time-averaged results using the quasi-1D solver in HPHall. Three test cases with a purely radial magnetic field matched the quasi-1D discharge current value to within 1\%, as expected because the Cartesian mesh aligns identically with the mesh from HPHall, thus allowing for a consistent comparison. For the cases with non-zero axial components of the magnetic field, we observed agreement within less than $10 \%$ with HPHall values (between $1.4 \%$ and $8.3 \%$ ). We observe larger discrepancies for the curved field cases that the purely radial cases due to the differences in the quasi-1D and 2D domain for a curved magnetic field, in particular near the cathode, where due to field line curvature the quasi-1D domain neglects several corner cells that are included in the $2 \mathrm{D}$ mesh. Lastly, the plume case generated a current that was $70 \%$ higher for the $2 \mathrm{D}$ model than quasi-1D due to the significant differences in the domain shape, as well as the cathode boundary condition that is imposed along a curved line in the quasi-1D case, versus vertically on the eastern cell interface for the 2D model.

The plasma potential and axial electric field spatial distributions for the channel test cases were presented, and good qualitative agreement was observed when compared to the quasi-1D results. A high value of electric field was observed at the cathode boundary that was not captured by the quasi-1D model, since this region was not included in the quasi-1D domain. The plasma potential distribution for the plume simulation was also presented and while the results are physically reasonable, a consistent comparison with the quasi-1D model can only be performed once the electron model is fully integrated in the hybrid-PIC framework. 
Next, the global Joule heating of the thruster and the heating power distribution were investigated. For the purely radial test cases we observed that the 2D model computed global heating values between $39 \%$ and $7.8 \%$ different than those from HPHall. It is shown that the main reason the results differ is that the quasi-1D model produces a null radial electron current for the cases with a purely radial magnetic field, while the 2D model gives a nonzero value. However, for the cases with a curved magnetic field the values obtained were not physical. It was shown that the difference in geometry between the quasi-1D domain and the 2D Cartesian mesh was responsible for the discrepancies observed, and that the differences are reduced with mesh refinement.

\section{Future Work}

The next step is to couple the 2D electron model with a heavy species solver and perform self-consistent simulations of the test cases shown in the present work. Furthermore, mesh refinement studies will be performed to further investigate the region of negative Joule power density that was observed near the cathode.

Additionally, the electron temperature solver may be improved for faster convergence by using a fully implicit method, which would guarantee unconditional stability. And for further improvements, a more sophisticated time integration scheme may be used, than the simple Euler forward differencing scheme that was implemented thus far.

Finally, the model will be applied to simulate a nested-channel Hall thruster to study the channel interaction and evaluate the location of the ionization region during single versus multiple channel operation.

\section{Acknowledgments}

This work was supported by a NASA Space Technology Research Fellowship, grant number: NNX13AL51H.

\section{References}

\footnotetext{
${ }^{1}$ Fife, J.M., "Hybrid-PIC Modeling and Electrostatic Probe Survey of Hall Thrusters," Ph.D. Dissertation, Dept. of Aeronautics and Astronautics, Massachusetts Institute of Technology, Cambridge, MA, 1998.

${ }^{2}$ Hofer, R.R., Mikellides, I.G., Katz, I., and Goebel, D.M., "BPT-4000 Hall Thruster Discharge Chamber Erosion Model Comparison with Qualification Life Test Data", 30th International Electric Propulsion Conference, IEPC-2007-267, Florence, Italy, September 17 - 20, 2007.

${ }^{3}$ Gamero-Castano, M. and Katz, I., "Estimation of Hall Thruster Erosion Using HPHall", 29th International Electric Propulsion Conference, IEPC-2005-303, Princeton University, Princeton, October 31 - November 4, 2005.

${ }^{4}$ Hofer, R.R., Mikellides, I.G., Katz, I., and Goebel, D.M., "Wall Sheath and Electron Mobility Modeling in Hybrid-PIC Hall Thruster Simulations", 43rd AIAA/ASME/SAE/ASEE Joint Propulsion Conference, AIAA 2007-5267, Cincinnati, OH, July $8-11,2007$.

${ }^{5}$ Hofer, R. R., et al., "Efficacy of Electron Mobility Models in Hybrid-PIC Hall Thruster Simulations," AIAA/ASME/SAE/ASEE Joint Propulsion Conference 85 Exhibit, AIAA 2008-4924, Hartford, CT, July 21-23, 2008.

${ }^{6}$ Hara, K., Sekerak, M. J., Boyd, I. D., and Gallimore, A. D., "Mode transition of a Hall thruster discharge plasma", Journal of Applied Physics, Vol. 115, Article 203304, 2014.

${ }^{7}$ Mikellides, I. G., et. al., "Magnetic Shielding of the Acceleration Channel Walls in a Long-Life Hall Thruster", AIAA/ASME/SAE/ASEE Joint Propulsion Conference 86 Exhibit, AIAA 2010-6942, Nashville, TN, July 25-28, 2010.

${ }^{8}$ Liang, R, "The Combination of Two Concentric Discharge Channels into a Nested Hall-Effect Thruster," Ph.D. Dissertation, Dept. of Aerospace Engineering, University of Michigan, Ann Arbor, MI, 2013.

${ }^{9}$ Florenz, R.E., "The X3 100-kW Class Nested-Channel Hall Thruster: Motivation, Implementation and Initial Performance," Ph.D. Dissertation, Aerospace Engineering Dept., University of Michigan., Ann Arbor, MI, 2014.

${ }^{10}$ Dragnea, H. C., Hara, K., and Boyd, I. D., "FULlY 2D NUMERICAL SIMULATION OF A NESTED CHANNEL HALL THRUSTER," Space Propulsion Conference, SP2016-3124969, Rome, Italy, May 2-6, 2016.

${ }^{11}$ Geng, J., Brieda, L. and Keidar, M., On applicability of the "thermalized potential" solver in simulations of the plasma flow in Hall thrusters, Journal of Applied Physics, Vol. 114, No. 10, 2013

$\checkmark{ }^{12} \mathrm{Koo}, \mathrm{J}$. W., et al., "2D electrostatic potential solver for Hall thruster simulation," AIAA/ASME/SAE/ASEE Joint Propulsion Conference 83 Exhibit, AIAA 2006-4832, Sacramento, CA, July 9-12, 2006.

${ }^{13}$ Mikellides, I. G., Katz, I., Hofer, R. R. and Goebel, D. M. Hall-Effect Thruster Simulations with 2-D Electron Transport and Hydrodynamic Ions, $31^{\text {st }}$ International Electric Propulsion Conference, 20 - 24 September 2009, Ann Arbor, MI
} 
${ }^{14}$ Goebel, D. M. and Katz, I. Fundamentals of Electric Propulsion: Ion and Hall Thrusters, Jet Propulsion Laboratory California Institute of Technology, Pasadena, CA, 2008 